\title{
ACTIVIDAD ANTIESTAFILOCÓCCICA Y ANTIBIOPELÍCULA DE LOS EXTRACTOS DE Juglans neotropica DIELS, Piper lineatum RUIZ\&PAV. Y Terminalia catappa L.
}

\section{Antistaphyloccocal activity and antibiofilm activity of the extracts of Juglans neotropica Diels, Piper lineatum Ruiz \& Pav. and Terminalia catappa L.}

Julio R Ruiz ${ }^{1}$, Mirtha Roque ${ }^{1}$, María E Salazar ${ }^{1}$, Gerardo Gamarra $^{1}$, Gladys C Arias ${ }^{2}$, Delia Y Whu ${ }^{1}$, Emma M Acosta ${ }^{1}$, José Irey $^{1}$, Luz F Guadalupe ${ }^{1}$, José A Llahuilla², José F Chávez ${ }^{3}$, Deyvis D Solis ${ }^{3}$, Goannie J Basualdo ${ }^{3}$, Oscar G Santa Cruz ${ }^{3}$

${ }^{1}$ Instituto de Investigación en Química Biológica, Microbiología y Biotecnología "Marco Antonio Garrido Malo"; ${ }^{2}$ Instituto de Investigaciones en Ciencias Farmacéuticas y Recursos Naturales "Juan de Dios Guevara"; ${ }^{3}$ Estudiante de Pregrado. UNMSM

\section{RESUMEN}

En este estudio, se investigó la actividad antiestafilocóccica in-vitro de los extractos etanólicos e hidroalcohólicos de 3 plantas medicinalesperuanas:Juglans neotropica Diels(corteza), Piperlineatum Ruiz\& Pav. (hojas)y Terminalia catappaL. (hojas); recolectadas en las regiones de Amazonas y Cajamarca, en el Perú. La actividad antiestafilocóccica se evaluó mediante el método de microdilución. Los microorganismos utilizados fueron las bacterias Staphylococcus aureus ATCC 25923 y Staphylococcus epidermidis ATCC 12228. Los extractos investigados presentaron actividad significativa frente a ambas bacterias, con una Concentración Mínima Inhibitoria (CMI) de 125 a 500 Mg/mL para Staphylococcus aureus, teniendo mayor actividad el extracto etanólico de Juglans neotropica Diels, y de 250 a $500 \mu \mathrm{g} / \mathrm{mL}$ para Staphylococcus epidermidis, teniendo mayor actividad el extracto hidroalcohólico de Piper lineatum Ruiz \& Pav. El análisis fitoquímico confirmó la presencia de compuestos terpénicos, flavonoides y alcaloides en los 6 extractos estudiados. La determinación de la Concentración Mínima Inhibitoria de Biopelícula (CMIB) se efectuó por el método de microdilución en placa modificado, utilizando cepas clínicas de Staphylococcus aureus y Staphylococcus epidermidis productoras de biopelícula. Solamente los extractos etanólicos e hidroalcohólicos de las hojas de Piper lineatum L. poseen actividad significativa con CMIB de 500 $\mu$ g/mL para ambas cepas. Los extractos etanólicos e hidroalcohólicos de Piper lineatum L. pueden ser buenos candidatos para la búsqueda de metabolitos que sirvan para combatir infecciones asociadas a biopelículas de Staphylococcus aureus y Staphylococcus epidermidis.

Palabras clave: Actividad antiestafilocóccica, actividad antibiopelícula, Piper lineatum, Juglans neotropica, Terminalia catappa.

\section{SUMMARY}

In this study, was investigated the antistaphylococcal activity in vitro of ethanol and hydroalcoholic extracts of 3 peruvian medicinal plants: Juglans neotropica Diels (bark), Piper lineatum Ruiz \& Pav. (leaves) and Terminalia catappa L. (leaves). The species were collected in the regions of Amazonas and Cajamarca, in Peru. Antistaphylococcal activity was assessed by microdilution method. The organisms used were bacteria Staphylococcus aureus ATCC 25923 and Staphylococcus epidermidis ATCC 12228. All extracts investigated showed significant activity against both bacteria with a minimum inhibitory concentration (MIC) of 125 to 500 $\mu \mathrm{g} / \mathrm{mL}$ for Staphylococcus aureus, with increased activity of the ethanol extract of Juglans neotropica Diels, and of 250 to 500 gg/ $\mathrm{mL}$ for Staphylococcus epidermidis, having greater activity the hydroalcoholic extract of Piper lineatum Ruiz \& Pav. Phytochemical analysis confirmed the presence of terpene compounds, flavonoids and alkaloids on 6 extracts studied. The determination of Minimum Inhibitory Concentration of Biofilm (MICB) was performed by the plate microdilution method modified, using clinical isolates of Staphylococcus aureus and Staphylococcus epidermidis biofilm producing. Only hydroalcoholic and ethanolic extracts of leaves of Piper lineatum L. have significant activity with CMIB $500 \mu \mathrm{g} / \mathrm{mL}$ for both strains. The ethanol and hydroalcoholic extracts of Piper lineatum L may be good candidates to search of metabolites useful to combat biofilm-associated infections by Staphylococcus aureus and Staphylococcus epidermidis.

Keywords: Antistaphylococcal activity, anti-biofilm activity, Piper lineatum, Juglans neotropica, Terminalia catappa.

\section{INTRODUCCIÓN}

as enfermedades infecciosas representan un problema crítico de salud y una de las principales causas de morbilidad y mortalidad, a nivel mundial. En la naturaleza, la mayoría de los microorganismos viven en grupos formados por muchedumbres, que se adhieren a una superficie, este tipo de comunidades se conoce como biopelícula ${ }^{(1)}$. La biopelícula es uno de los factores de virulencia más importantes de los estafilococos y juega un papel en muchas infecciones tales como la endocarditis de válvula nativa, otitis media, infecciones 
del tracto urinario, fibrosis quística, artritis séptica aguda y otras relacionadas con dispositivos médicos ${ }^{(2)}$.

Es ampliamente conocido que el Perú es uno de los países megadiversos del mundo y posee una amplia cultura con respecto al uso de las plantas medicinales. La Amazonía peruana es una de las zonas del planeta con mayor diversidad vegetal, de la cual menos del $1 \%$ ha sido estudiada desde el punto de vista fitoquímico y farmacológico, por lo que su estudio podría conducir al descubrimiento de un gran número de nuevas moléculas bioactivas con posible aplicación terapéutica ${ }^{(3)}$.

En este proyecto, se investigaron plantas medicinales peruanas con gran actividad antibacteriana, procedentes de las regiones Amazonas y Cajamarca, que podrían servir como candidatas para el desarrollo de agentes antimicrobianos y/o fitomedicamentos estandarizados.

En el Perú, Juglans neotropica Diels (Nogal) se encuentra entre 1000 y $3000 \mathrm{msnm}$, en las regiones de Amazonas, Cusco, Huánuco, Junín, Lima, Loreto, San Martín, Ucayali ${ }^{(4)}$. En lo que respecta a este género, Quave y col., demostraron que Juglans regia tiene actividad antibiopelícula contra Staphylococcus aureus meticilina resistente con una $\mathrm{CI}_{50}<32 \mu \mathrm{g} / \mathrm{mL}^{(5)}$.

Se calcula que en nuestro país existen aproximadamente 811 especies de la familia Piperaceae, repartidas en 3 géneros, de las cuales 528 son nativas ${ }^{(6)}$. Entre ellas encontramos a Piper lineatum Ruiz \& Pav. Marçal y col., han demostrado que el extracto de acetato de etilo de las hojas Piper regnellii, posee CMI de 16 $\mu \mathrm{g} / \mathrm{mL}$ contra Staphylococcus aureus meticilina resistente ${ }^{(7)}$.

Terminalia catappa L. (Castañilla) se encuentra en la selva peruana, se ha reportado que el extracto etanólico de sus hojas tiene actividad contra bacterias Gram positivas, entre ellas Staphylococcus aureusy Staphylococcus epidermidis ${ }^{(8-9)}$, y bacterias Gram negativas ${ }^{(8)}$.

Los objetivos de la investigación fueron:determinarlaCMIylaCMIBdelos extractos etanólicos e hidroalcohólicos de las plantas seleccionadas, mediante la prueba de microdilución en placa contra Staphylococcus aureus y Staphylococcus epidermidis.

Rf: Ratio of front

\section{MATERIAL Y MÉTODOS}

Las plantas que se describen en la tabla 1 fueron recolectadas en las regiones de Amazonas y Cajamarca. El material recolectado fue acondicionado y estabilizado en el Laboratorio de Microbiología de la Facultad de Farmacia y Bioquímica de la Universidad Nacional Mayor de San Marcos. Una vez secada la muestra se sometió a un proceso de reducción de tamaño de partículas en un molino de cuchillas $\mathrm{N}^{\circ} 21$.

\section{Preparación de los extractos}

Se trabajó con la droga en polvo de las especies elegidas (tabla 1). La extracción etanólica se realizó por maceración a temperatura ambiente con etanol al 95\%, utilizando la proporción de 1:10 P/V por 72 h. Para los extractos hidroalcohólicos se utilizó la misma proporción del preparado etanólico, con la diferencia que el solvente de extracción fue etanol:agua (70:30). Los extractos obtenidos se filtraron, rota-evaporaron y conservados en refrigeración ${ }^{(5)}$. Para los bioensayos, los extractos fueron resuspendidos a una concentración de $10 \mathrm{mg} / \mathrm{mL}$, diluídos en dimetilsulfóxido (DMSO), esterilizados por filtración $(0,2 \mu \mathrm{m})$ y almacenados a $4^{\circ} \mathrm{C}$.

\section{Análisis fitoquímico preliminar}

Los extractos fueron sometidos a cromatografía en capa fina en placas (GF 25460; Merck) 250 mm deespesor;

Tabla 1. Plantas medicinales seleccionadas para el ensayo antimicrobiano.

\begin{tabular}{lccc}
\hline \multicolumn{1}{c}{ Especie } & Familia & $\begin{array}{c}\text { Nombre } \\
\text { común }\end{array}$ & $\begin{array}{c}\text { Parte } \\
\text { usada }\end{array}$ \\
\hline $\begin{array}{l}\text { Juglans neotropica Diels } \\
\text { Piper lineatum } \text { Ruiz \& Pav. }\end{array}$ & Juglandaceae & Nogal & Corteza \\
Terminalia catappa L. & Combretaceae & Luto & Hojas \\
\hline
\end{tabular}

Tabla 2. Análisis fitoquímico preliminar por cromatografía de capa fina.

\begin{tabular}{|c|c|c|c|c|}
\hline \multirow{2}{*}{ Muestras } & \multicolumn{4}{|c|}{ Rf de Compuestos } \\
\hline & Alcaloides & Flavonoides & Cumarinas & Terpenos \\
\hline $\begin{array}{l}\text { Extracto etanólico de } \\
\text { Juglans neotropica Diels }\end{array}$ & & $\begin{array}{c}0,7894 \\
0,6842 \\
0,421\end{array}$ & & 0,4 \\
\hline $\begin{array}{l}\text { Extracto etanólico de } \\
\text { Piper lineatum Ruiz \& Pav. }\end{array}$ & 0,741 & $\begin{array}{c}0,8 \\
0,5764 \\
0,411\end{array}$ & 0,75 & $\begin{array}{l}0,9058 \\
0,7294 \\
0,5882\end{array}$ \\
\hline $\begin{array}{l}\text { Extracto etanólico de } \\
\text { Terminalia catappa L. }\end{array}$ & 0,6364 & 0,1515 & 0,2424 & 0,7575 \\
\hline $\begin{array}{l}\text { Extracto hidroalcohólico de } \\
\text { Juglans neotropica Diels }\end{array}$ & & $\begin{array}{l}0,4421 \\
0,8631 \\
0,9263\end{array}$ & & 0,2421 \\
\hline $\begin{array}{l}\text { Extracto hidroalcohólico de } \\
\text { Piper lineatum Ruiz \& Pav. }\end{array}$ & & $\begin{array}{c}0,3678 \\
0,7471 \\
0,885 \\
0,931\end{array}$ & & $\begin{array}{c}0,908 \\
0,7471 \\
0,5862\end{array}$ \\
\hline $\begin{array}{l}\text { Extracto hidroalcohólico de } \\
\text { Terminalia catappa L. }\end{array}$ & 0,606 & 0,09 & 0,6263 & 0,8 \\
\hline
\end{tabular}


fueron desarrollados con cloroformo:metanol:agua (80:18:2), que permitió la separación de componentes en un amplio rango de valores de Rf. Los componentes fueron visualizados bajo luz UV (254 y $366 \mathrm{jm}$ ) y revelados con los reactivos de Dragendorff modificado, hidróxido de potasio metanólico, cloruro de aluminio y anisaldehído/ácido sulfúrico, para alcaloides, cumarinas, flavonoides y terpenos, respectivamente ${ }^{(10)}$.

\section{Bacterias y condiciones de cultivo}

Se usaron las cepas de Staphylococcus aureus ATCC 25923 y Staphylococcus epidermidis ATCC 12228 para la determinación de la CMI; así como Staphylococcus aureus y Staphylococcus epidermidis meticilino resistentes productores de biopelículas para la determinación de la CMIB. Las bacterias fueron sembradas en placas de agar tripticasa soya (Merck) e incubadas a $37^{\circ} \mathrm{C}$ por $18-24 \mathrm{~h}$. Para preparar el inóculo base se resuspendieron colonias en solución salina estéril al o,85\% y se ajustó a la turbidez equivalente al tubo 0,5 de la escala de McFarland, que sirvió para determinar la $\mathrm{CMI}$ y la $\mathrm{CMIB}{ }^{(5)}$. Se realizaron las diluciones necesarias con caldo Mueller Hinton (Merck) cuando el ensayo lo requirió.

\section{Determinación de la concentración mínima inhibitoria (CMI)}

Se realizó de acuerdo al método de Sarker y col. (11), pero con modificaciones. El ensayo usó placas de poliestireno de 96 pozos de fondo plano y estériles (Brand), caldo Mueller Hinton (Merck) y resazurina (Sigma) como marcador de células vivas. Los extractos se prepararon a concentraciones de $7,81-4000 \mu \mathrm{g} /$ $\mathrm{mL}$ y el control vancomicina de $8-1024 \mu \mathrm{g} / \mathrm{mL}$ en diluciones dobles seriadas, utilizando como diluyente final caldo Mueller Hinton, que representaron el doble de concentración final deseada. Se incluyeron pozos de control de crecimiento (bacterias más caldo Mueller Hinton) y control deesterilidad (caldo Mueller Hinton). Todas las pruebas fueron realizadas por triplicado. Las diluciones de los extractos se dispensaron en los pozos de las placas de microdilución a razón de $100 \mathrm{uL}$ de cada una de las diluciones. Luego se adicionó a cada pozo $100 \mu \mathrm{L}$ del inóculo $2 x$ que ya incluía la solución indicadora de resazurina (de acuerdo al método de Liu y col. $\left.{ }^{(12)}\right)$, para alcanzar el inóculo final de $5 \times 10^{5}$ $\mathrm{ufc} / \mathrm{mL}^{(11)}$. Las placas se llevaron a incubación a $37^{\circ} \mathrm{C}$ por 18-24 h. El cambio de color se evaluó visualmente. Cualquier cambio de color de púrpura a rosado se registró como positivo. La concentración más baja en la que no se produjo cambio de color se tomó como el valor de CMI ${ }^{(11)}$.

\section{Comprobación de la formación de biopelículas}

Se evaluó la habilidad de formar biopelículas de las cepas de estudio por el método de Freeman y col. ${ }^{(13)}$.

\section{Determinación de la concentración mínima inhibitoria de biopelícula (CMIB)}

El métododeAntunesycol. ${ }^{(14)}$, con modificaciones, consiste en evaluar el efecto de los extractos sobre biopelículas formadas en microplacas estériles de 96 pozos, de fondo plano (Brand); en las que a cada pozo se agregaron $100 \mu \mathrm{L}$ del inóculo $\left(5 \times 10^{5} \mathrm{ufc} / \mathrm{mL}\right)$ de cepas de Staphylococcus aureus y Staphylococcus epidermidis productoras de biopelícul a. Como control de esterilidad se usó una columna con sólo caldo Mueller Hinton (control de esterilidad), incubándose por $24 \mathrm{~h}$ a $37^{\circ} \mathrm{C}$. Posteriormente se procedió a retirar el contenido de los pozos y agregar $100 \mu \mathrm{L}$ de las diluciones de los extractos $(3,91-2000 \mu \mathrm{g} / \mathrm{mL})$ y vancomicina $(4-512 \mu \mathrm{g} /$ $\mathrm{mL}$ ). Seguidamente se reincubaron las microplacas por $24 \mathrm{~h}$ a $37^{\circ} \mathrm{C}$, se retiraron los contenidos de los pozos y enjuagaron 3 veces con solución salina fisiológica estéril. Luego se secaron los pozos a $37^{\circ} \mathrm{C}$ y se les agregó $100 \mu \mathrm{L}$ de caldo Mueller Hinton con resazurina. Se incubaron las microplacas de $30-60 \mathrm{~min}$ a $37^{\circ} \mathrm{C}$ y se procedió a su lectura visual. Cualquier cambio de color de púrpura a rosado se registró como positivo. La concentración más baja a la que no presentó cambio de color se tomó como el valor de la CMIB $^{(12,14)}$.

\section{RESULTADOS}

\section{Análisis Fitoquímico Preliminar}

El análisis fitoquímico preliminar reveló la presencia de alcaloides, flavonoides y terpenos en los 6 extractos estudiados: extractos etanólicos e hidroalcohólicos de Juglans neotropica Diels, Piper lineatum y Terminalia catappa. Los valores de frente de solventes Rf (Ratio of front) de las 6 muestras se presentan en la tabla 2.

\section{Determinación de la Concentración Mínima Inhibitoria \\ Todos los extractos tuvieron una CMI $<1000 \mu \mathrm{g} /$ $\mathrm{mL}$, la cual es significativa según los criterios de Holetz y col. ${ }^{(15)}$. Los resultados se muestran en la tabla 3 y en la figura 1.}

\section{Determinación de la Concentración Mínima Inhibitoria de Biopelícula (CMIB) \\ Los resultados de los ensayos se muestran en la tabla 3.}


Tabla 3. Actividad antiestafilocóccica y antibiopelícula de los extractos crudos.

\begin{tabular}{lcccccc}
\hline \multirow{2}{*}{$\begin{array}{c}\text { Especies de } \\
\text { plantas }\end{array}$} & $\begin{array}{c}\text { Partes de } \\
\text { las plantas } \\
\text { ensayadas }^{\text {a }}\end{array}$ & $\begin{array}{c}\text { Tipo } \\
\text { de } \\
\text { extractos }\end{array}$ & \multicolumn{2}{c}{ CMI $(\mu \mathrm{g} / \mathrm{mL})$} & \multicolumn{2}{c}{ CMIB $(\mu \mathrm{g} / \mathrm{mL})$} \\
\hline \multirow{2}{*}{ Juglans neotropica } & $\mathrm{C}$ & $\mathrm{E}$ & 125 & 125 & 2000 & 1000 \\
& $\mathrm{C}$ & $\mathrm{HA}$ & 500 & 500 & 2000 & 250 \\
Piper lineatum & $\mathrm{H}$ & $\mathrm{E}$ & 250 & 250 & 500 & 250 \\
& $\mathrm{H}$ & $\mathrm{HA}$ & 500 & 250 & 500 & 500 \\
Terminalia catappa & $\mathrm{H}$ & $\mathrm{E}$ & 250 & 250 & $>2000$ & 2000 \\
Control & $\mathrm{H}$ & $\mathrm{HA}$ & 500 & 500 & $>2000$ & 2000 \\
Vancomicina & & & & & & ${ }^{*}$ \\
\hline
\end{tabular}

(a) Partes de las plantas ensayadas: $\mathrm{C}$, corteza; $\mathrm{H}$, hojas $\mathrm{CMI}$ : concentración mínima inhibitoria

(b) Tipo de extractos: E, etanólico; HA, hidroalcohólico CMIB: concentración mínima inhibitoria de biopelícula

S.a.: Staphylococcus aureus ATCC 25923, S.e.: Staphylococcus epidermidis ATCC 12228

* Productora de biopelícula

\section{DISCUSIÓN}

Los extractos etanólico e hidroalcohólico de la corteza de Juglans neotropica Diels poseen muy buena actividad contra las cepas de Staphylococcus aureus ATCC 25923 y Staphylococcus epidermidis ATCC 12228, con CMIs de $125 \mu \mathrm{g} / \mathrm{mL}$ y $500 \mu \mathrm{g} / \mathrm{mL}$. Estos resultados concuerdan con los estudios realizados por Ruiz \& Roque ${ }^{(9)}$ y Lopez y col. ${ }^{(16)}$, que en sus estudios sólo demostraron la actividad contra Staphylococcus aureus por métodos de difusión. También se ha reportado que el aceite esencial de hojas de Juglans regia tiene buena actividad contra Staphylococcus aureus y Staphylococcus epidermidis ${ }^{(17)}$. Por otro lado Oliveira y col. ${ }^{(18)}$ encontraron CMI de $100 \mu \mathrm{g} / \mathrm{mL}$ contra Staphylococcus aureus con el extracto acuoso de la nuez de Juglans regia, siendo este valor cercano al encontrado en la presente investigación.

El análisis fitoquímico reporta la presencia de flavonoides y terpenos en ambos extractos de la corteza de Juglans neotropica Diels; estos compuestos podrían explicar su actividad antiestafilocóccica ${ }^{(19)}$. En cuanto a la actividad anti-biopelícula, ambos extractos son inactivos frente a la biopelícula de Staphylococcus aureus, con valores de 4 a 5 veces de su CMI. Sin embargo, los extractos etanólico e hidroalcohólico tienen CMIB de $1000 \mu \mathrm{g} / \mathrm{mL}$ y $250 \mu \mathrm{g} / \mathrm{mL}$, respectivamente, contra la biopelícula de Staphylococcus epidermidis, siendo este último valor la mitad del encontrado para su CMI. La literatura reporta que los frutos inmaduros de Juglans regia tienen una buena actividad anti-biopelícula con una concentración inhibitoria de formación de biopelícula de Staphylococcus aureus al 50\% de $16 \mu \mathrm{g} / \mathrm{mL}^{(5)}$.

Los extractos etanólico e hidroalcohólico de las hojas de Piper lineatum L. mostraron actividad significativa contra Staphylococcus aureus, Staphylococcus epidermidis con CMI de $250 \mu \mathrm{g} / \mathrm{mL}$, lo que se cataloga como de mediana actividad (16). Estos resultados concuerdan con los encontrados por Ruiz \& Roque con el método de difusión en agar ${ }^{(9)} \mathrm{y}$ con los hallazgos de De la Cruz ${ }^{(20)}$.

El género Piper es conocido por su actividad antimicrobiana. Se han observado resultados similares, en cuanto a la actividad antibacteriana contra Gram positivos, del extracto de partes aéreas de Piper aduncum ${ }^{(8)}$ y del extracto en acetato de etilo de las hojas de Piper regnellii ${ }^{(21)}$.

El análisis fitoquímico preliminar reveló la presencia de alcaloides, cumarinas, flavonoides y terpenos en el extracto etanólico de las hojas de Piper lineatum L., y de sólo flavonoides y terpenos en su extracto hidroalcohólico; estos compuestos fitoquímicos podrían explicar su actividad antiestafilocóccica ${ }^{(19)}$. Los resultados de la CMIB de los extractos etanólicos e hidroalcohólicos de las hojas de esta planta, son de $500 \mu \mathrm{g} / \mathrm{mL}$ para biopelículas de Staphylococcus aureus, siendo apenas 2 veces la CMI en el caso del extracto etanólico y manteniéndose el mismo valor que el CMI en el extracto hidroalcohólico.

Respecto a los valores de CMIB contra las biopelículas de Staphylococcus epidermidis, estos fueron de $250 \mu \mathrm{g} / \mathrm{mL}$ y $500 \mu \mathrm{g} / \mathrm{mL}$, para el extracto etanólico e hidroalcohólico, respectivamente. Estos valores casi no varían de su CMI. Esta planta debe ser investigada con mayor profundidad, debido a los excelentes resultados encontrados en la presente investigación.

Los extractos etanólico e hidroalcohólico de las hojas de Terminalia catappa mostraron actividad antiestafilocóccica significativa contra ambos Staphylococcus, con una CMI de $250 \mu \mathrm{g} / \mathrm{mL}$ del extracto etanólico para ambas cepas y de $500 \mu \mathrm{g} / \mathrm{mL}$ del extracto hidroalcohólico. Estos resultados concuerdan con los reportados en otros estudios ${ }^{(8,22)}$.

Otras especies del género también reportan actividad antiestafilocóccica, por ejemplo los extractos de la corteza del tallo y la raíz de Terminalia chebula y Terminalia bellerica ${ }^{(23)}$. El análisis 


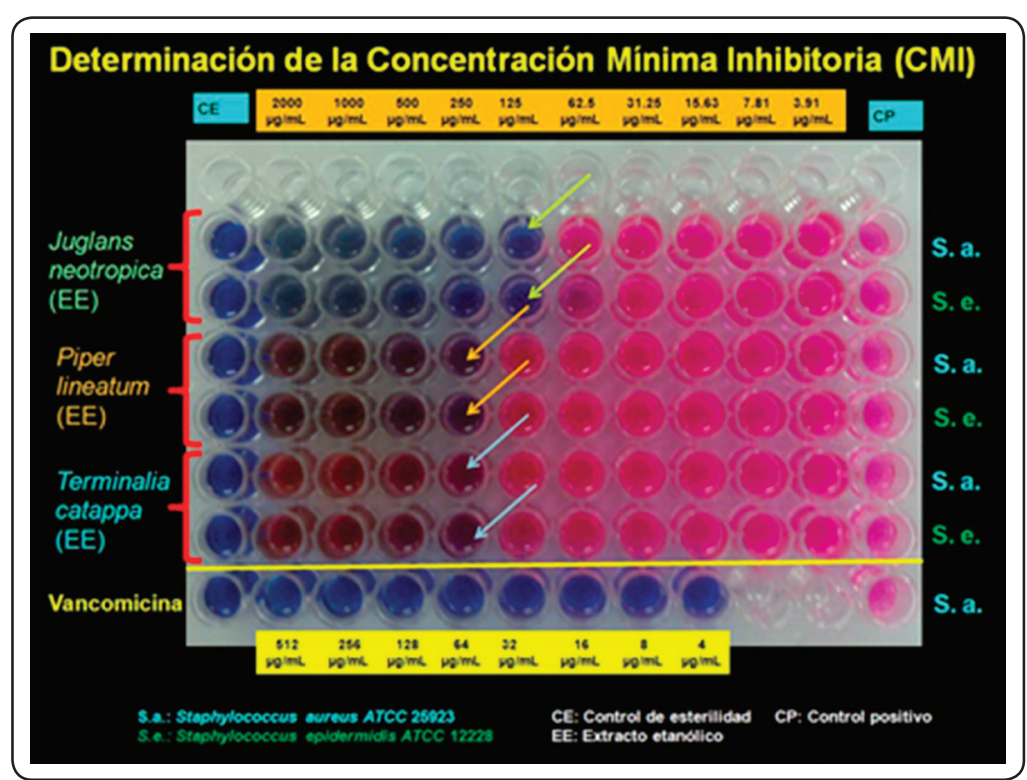

Figura 1. Determinación de la Concentración Mínima Inhibitoria.

fitoquímico preliminar reveló la presencia, en ambos extractos de Terminalia catappa L., de alcaloides, cumarinas, flavonoides y terpenos, aunque en menor cantidad a los hallados en Piper lineatum Ruiz \& Pav.; estos metabolitos pueden explicar su acción antibacteriana ${ }^{(19)}$. Tabopta y col., reportaron que un derivado triterpénico de Terminalia superba posee actividad antibacteriana (24). Los extractos de Terminalia catappa L. no tuvieron actividad significativa contra las biopelículas de Staphylococcus aureus y Staphylococcus epidermidis, reportando CMIB $\geq 2000 \mu \mathrm{g} / \mathrm{mL}$.

\section{CONCLUSIONES}

Los extractos etanólicos de la corteza de Juglans neotropica Diels, de hojas de Piper lineatum Ruiz \& Pav. y hojas de Terminalia catappa L.; presentaron CMI de $125 \mu \mathrm{g} / \mathrm{mL}, 250 \mu \mathrm{g} / \mathrm{mL}$ y $250 \mu \mathrm{g} / \mathrm{mL}$, respectivamente, contra Staphylococcus aureus ATCC 25923; y sus extractos hidroalcohólicos, CMI de $500 \mu \mathrm{g} / \mathrm{mL}$, contra el mismo microorganismo.

Los extractos hidroalcohólicos de la corteza de Juglans neotropica Diels, de hojas de Piper lineatum Ruiz \& Pav. y hojas de Terminalia catappa L.; presentaron CMI de $125 \mu \mathrm{g} / \mathrm{mL}, 250 \mu \mathrm{g} / \mathrm{mLy} 250 \mu \mathrm{g} / \mathrm{mL}$, respectivamente, contra Staphylococcus epidermidis ATCC 12228; y sus extractos hidroalcohólicos presentaron una CMI de $500 \mu \mathrm{g} / \mathrm{mL}$ contra el mismo microorganismo, a excepción del obtenido de Piper lineatum Ruiz \& Pav. que presentó CMI de 250 Hg/mL.
Los extractos etanólicos e hidroalcohólicos de hojas de Terminalia catappa L. presentaron una CMIB > $2000 \mu \mathrm{g} / \mathrm{mL}$, los extractos etanólicos e hidroalcohólicos de corteza de Juglans neotropica Diels presentaron CMIB de $2000 \mu \mathrm{g} / \mathrm{mL}$, y los extractos etanólicos e hidroalcohólicos de hojas de Piper lineatum presentaron una CMIB de $500 \mu \mathrm{g} / \mathrm{mL}$ frente a biopelículas de Staphylococcus aureus meticilino resistentes.

Los extractos etanólicos e hidroalcohólicos de las hojas de Terminalia catappa L. presentaron CMIB de $2000 \mu \mathrm{g} /$ $\mathrm{mL}$ y los de la corteza de Juglans neotropica Diels CMIB de $1000 \mu \mathrm{g} / \mathrm{mL}$ y $250 \mu \mathrm{g} / \mathrm{mL}$, respectivamente; y los de las hojas de Piper lineatum Ruiz \& Pav. mostraron CMIB de 250 $\mu \mathrm{g} / \mathrm{mL}$ y de $500 \mu \mathrm{g} / \mathrm{mL}$, respectivamente, frente a biopelículas de Staphylococcus epidermidis meticilino resistentes.

Agradecimiento: Proyecto financiado por el VRIUNMSM, código de proyecto № 120403061.

\section{REFERENCIAS BIBLIOGRÁFICAS}

1. Donlan RM, Costerton JW. Biofilms: survival mechanisms of clinically relevant microorganisms. Clin Microbiol Rev 2002; 15(2): 167-93.

2. Agarwal A, Singh KP, Jain A. Medical significance and management of staphylococcal biofilm. FEMS Immunol Med Microbiol 2010; 58(2): 147-6o.

3. Desmachelier C, Witting F. Sesenta plantas medicinales de la Amazonía Peruana. Ecología, Etnomedicina y Bioactividad. Gráfica Bellido SRL. Lima, 2000.

4. Macbride JF. Juglandaceae, Flora of Perú Field Museum of Natural History, Botanical Series Collation 1943; 13(2/2): 263-6.

5. Quave CL, Plano LR, Pantuso T, Bennett BC. Effects of extracts from Italian medicinal plants on planktonic growth, biofilm formation and adherence of methicillinresistant Staphylococcus aureus. J Ethnopharmacol 2008; 118(3): 418-28.

6. Brack Egg A. Diccionario Enciclopédico de plantas útiles del Perú. Centro Bartolomé de las Casas. Cusco, 1999.

7. Marçal FJ, Cortez DA, Ueda-Nakamura T, Nakamura CV, Dias Filho BP. Activity of the extracts and neolignans from Piper regnellii against methicillin-resistant Staphylococcus aureus (MRSA). Molecules 2010; 24; 15(4): 2060-9.

8. Kloucek P, Polesny Z, Svobodova B, Vlkova E, Kokoska L. Antibacterial screening of some Peruvian medicinal plants used in Callería District. J Ethnopharmacol 2005; 99(2): 309-12. 
9. Ruiz J, Roque M. Actividad antimicrobiana de cuatro plantas del nor-oriente peruano. Ciencia e Investigación 2009; $12(1)$ : 41-7.

10. Nostro A, Germanò MP, D'angelo V, Marino A, Cannatelli MA. Extraction methods and bioautography for evaluation of medicinal plant antimicrobial activity. Lett Appl Microbiol 2000; 30(5): 379-84.

11. Sarker SD, Nahar L, Kumarasamy Y. Microtitre platebased antibacterial assay incorporating resazurin as an indicator of cell growth, and its application in the in vitro antibacterial screening of phytochemicals. Methods 2007; 42(4): 321-4.

12. Liu M, Seidel V, Katerere DR, Gray AI. Colorimetric broth microdilution method for the antifungal screening of plant extracts against yeasts. Methods. 2007; 42(4):325-9.

13. Freeman DJ, Falkiner FR, Keane CT. New method for detecting slime productionby coagulase negative staphylococci. J Clin Pathol 1989; 42(8): 872-4.

14. Antunes AL, Trentin DS, Bonfanti JW, Pinto CC, Perez LR, Macedo AJ, et al. Application of a feasible method for determination of biofilm antimicrobial susceptibility in staphylococci. APMIS 2010; 118(11): 873-7.

15. Holetz FB, Pessini GL, Sanches NR, Cortez DA, Nakamura $\mathrm{CV}$, Filho BP Screening of some plants used in the Brazilian folk medicine for the treatment of infectious diseases. Mem Inst Oswaldo Cruz 2002; 97(7): 1027-31.

16. Lopez A, Hudson JB, Towers GH. Antiviral and antimicrobial activities of Colombian medicinal plants. J Ethnopharmacol 2001; 77(2-3):189-96.

17. Rather MA, Dar BA, Dar MY, Wani BA, Shah WA, Bhat BA, et al. Chemical composition, antioxidant and antibacterial activities of the leaf essential oil of Juglans regia L. and its constituents. Phytomedicine 2012; 19(13): 1185-90.
18. Oliveira I, Sousa A, Ferreira IC, Bento A, Estevinho L, Pereira JA. Total phenols, antioxidant potential and antimicrobial activity of walnut (Juglans regia L.) green husks. Food Chem Toxicol 20o8; 46(7): 2326-31.

19. Ozçelik B, Kartal M, Orhan I. Cytotoxicity, antiviral and antimicrobial activities of alkaloids, flavonoids, and phenolic acids. Pharm Biol 2011; 49(4): 396-402.

20. De la Cruz J. Actividad antimicrobiana, antioxidante y determinación de la composición química mediante cromatografía de gases/espectrometría de masas (CG/EM) de los aceites esenciales de 3 especies de piper nativas del Perú. [Tesis para optar el título de Químico Farmacéutico]. Facultad de Farmacia y Bioquímica. UNMSM. Lima, 2012.

21. Pessini GL, Dias Filho BP, Nakamura CV, Cortez DA. Antibacterial activity of extracts and neolignans from Piper regnellii (Miq.) C. DC. var. pallescens (C. DC.) Yunck. Mem Inst Oswaldo Cruz. 2003; 98(8): 1115-20.

22. Shinde SL, Junne SB, Wadje SS. The diversity of antibacterial compounds of Terminalia species (Combretaceae). Pak J Biol Sci 2009; 12(22):1483-6.

23. Aqil F, Ahmad I. Antibacterial properties of traditionally used indian medicinal plants. Methods Find Exp Clin Pharmacol 2007; 29(2): 79-92.

24. Tabopda TK, Ngoupayo J, Khan Tanoli SA, Mitaine-Offer AC, Ngadjui BT, Ali MS, et al. Antimicrobial pentacyclic triterpenoids from Terminalia superba. Planta Med 2009; 75(5): 522-7.

\section{Correspondencia}

Nombre: $\quad$ Mg. Julio Reynaldo Ruiz Quiroz

Dirección: Jr. Puno 1002 -Lima 1 - Perú

E-mail:_ julioruizquiroz@gmail.com 